\title{
NEW WOLF-RAYET BINARIES
}

\author{
VIRPI S. NIEMELA ${ }^{1,2,3}$ \\ Instituto de Astronomía y Fisica del Espacio \\ CC67, Suc 28, 1428 Buenos Aires, Argentina
}

\begin{abstract}
Preliminary radial velocity orbits are presented for three binary systems containing Wolf-Rayet stars, namely Sk- $71^{\circ} 34$ in the LMC, and WR8 and WR98 in our galaxy. Sk- $71^{\circ} 34$ is found to be a WN3 + 06 double-lined binary with an elliptic orbit of period about 34 days. WR 8 and WR98 both have WN type spectra with carbon lines. In WR8 the $\mathrm{N}$ and $\mathrm{C}$ lines appear to move in antiphase, while in WR98 all emission lines have the same orbital motion.
\end{abstract}

\section{Introduction}

Radial velocity studies of stars with Wolf-Rayet spectra are needed for a sufficiently large sample of stars in order to ascertain the binary frequency among these stars. Further studies of binaries will give us fundamental information on the masses and mass limits of WolfRayet stars.

In this paper I present a radial velocity study of three stars with Wolf-Rayet spectra, all of which show velocity variations. Preliminary orbital parameters are also presented.

\section{Observations}

The observations were carried out at the Cerro Tololo Interamerican Observatory, Chile, with the Cassegrain spectrograph with IT attached to the $1 \mathrm{~m}$ Yale telescope. All spectrograms have a reciprocal dispersion of $45 \AA / \mathrm{mm}$. Spectra obtained before 1985, December, were recorded on photographic plates; thereafter on a CCD thru 3 images tubes.

The photographic spectra were measured for determination of radial velocities with the Grant oscilloscope engine at IAFE, Buenos Aires. The digital spectra were processed with IRAF at CTIO, La Serena.

\footnotetext{
${ }^{1}$ Senior Visitor, ESO, La Silla Observatory

${ }^{2}$ Member of Carrera del Investigador, CIC, Prov. Buenos Aires, Argentina

${ }^{3}$ Visiting Astronomer, CTIO, NOAO operated by AURA, Inc. under contract with the NSF
} 


\section{Radial Velocity Orbits}

\subsection{SK $-71^{\circ} 34$ :}

This hot star in the Large Magellanic Cloud has been classified by Conti and Garmany (1983) as WN3+O or O4f/WN3, depending whether the star may be a binary or not. I have obtained a total of 32 spectrograms, 9 photographic and 23 digital, of this star. The radial velocity of both absorption and emission lines is variable, and they show antiphased variations.

A period search routine applied to the observed radial velocities yields a period of 33.95 days, but other periods close to this cannot be excluded due to the limited data sample. Table 1 lists the orbital parameters of SK- $71^{\circ} 34$, and Figure 1 shows the observed radial velocities folded in the period of 33.95 days. The radial velocities of HeII and NV emissions show much scatter, therefore only the amplitude of their variation was considered.

\begin{tabular}{|c|c|c|c|}
\hline & & mean abs. & NV em. \\
\hline Period & (days) & 33.95 & \\
\hline e & & 0.41 & \\
\hline Vo & $(\mathrm{km} / \mathrm{s})$ & 189 & 219 \\
\hline $\mathrm{K}$ & $(\mathrm{km} / \mathrm{s})$ & 100 & 157 \\
\hline$\omega$ & $(\mathrm{deg})$ & 41 & \\
\hline Eo & $(2,440,000+)$ & 6448.54 & \\
\hline$a \sin i$ & $\left(\mathrm{R}_{\odot}\right)$ & 60.8 & 95.5 \\
\hline$M \sin ^{3} i$ & $\left(\mathrm{M}_{\odot}\right)$ & 27.6 & 17.6 \\
\hline
\end{tabular}
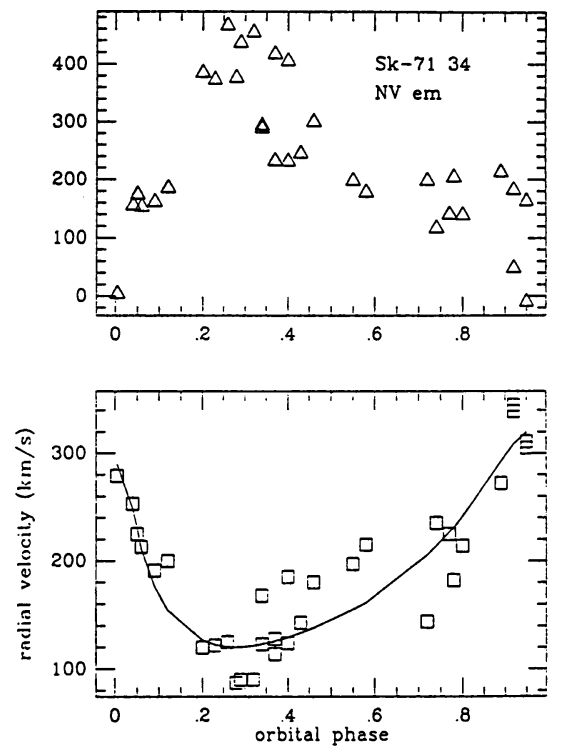

Figure 1. Observed radial velocities of mean absorption and NV emission in the spectrum of SK-71 31 . The curve represents the orbit from Table 1. 


\subsection{WR $8=$ HD 62910 :}

This WR star has a WN6 type spectrum with carbon lines, it has been classified as WN6+WC4 (cf.van der Hucht et al.1981). A previous radial velocity study (Niemela 1982) showed large amplitude variations of the blue-shifted HeI 3888 absorption, and smaller amplitud velocity variations of CIV 4441 and NIV emissions with much scatter.

Present data, based on 73 photographic spectrograms, confirm the previous variations. However, several periods are still possible, the shortest one being of 38.4 days. Longer periods, of about 55 and 64 days, are equally probable, and they produce elliptic orbits. The blue-shifted absorption of HeI 3888 and CIV 4441 emission seem to move in phase, but the amplitude of the velocity variations of the absorption is much larger than that of the emission.

The NV 4603 and NIV 4057 emissions apparently follow an orbital motion opposite to the HeI 3888 absorption, as shown in Figure 2. Table 2 lists the circular orbital elements for the period of 38.4 days. These elements should be taken only as an exersice, until the true period of HD 62910 will be firmly established.

TABLE 2. Circular orbital element of HD 62910

\begin{tabular}{lcrrr}
\hline element & & HeI abs. & CIV em. & NV em. \\
& & 3888.65 & 4441.03 & 4603.73 \\
\hline period & (days) & 38.4 & & \\
Vo & $(\mathrm{km} / \mathrm{s})$ & -1238 & 65 & 135 \\
$\mathrm{~K}$ & $(\mathrm{~km} / \mathrm{s})$ & 250 & 72 & 23 \\
Eo & $(2,440,000+)$ & 4659.6 & 4659.5 & 4639.0 \\
a sin i & $\left(\mathrm{R}_{\odot}\right)$ & 188.6 & 54.3 & 17.3 \\
$\mathrm{M} \sin ^{3} \mathrm{i}$ & $\left(\mathrm{M}_{\odot}\right)$ & & 0.8 & 2.6 \\
\hline
\end{tabular}

\subsection{WR $98=$ HDE 318016 :}

The spectrum of this star has been classified as WN7+WC7. No previous radial velocity studies of WR 98 has been published. From 41 photographic spectrograms obtained for the present study, the radial velocity of WR 98 was found to be variable from one epoch to another. A period search routine applied to the observed radial velocities yields as best period 47.8 days. Other periods close to this value are also possible. Table 3 lists the preliminary orbital elements of WR 98, and Figure 3 shows the observed radial velocities together with the orbits calculated with the elements from Table 3. Within the errors, the CIII-IV 4648 and HeII 4685 emissions show the same orbital motion. The NV 4603 and NIV 4057 emission lines also move in phase with the $\mathrm{C}$ and He emissions, but their radial velocities show much more scatter.

TABLE 3. Circular orbital elements of WR 98

\begin{tabular}{lcrrrr}
\hline element & & HeIle & CIII-IVe & NVe & NIVe \\
& & 4685 & 4647 & 4603 & 4057 \\
\hline period & $($ days $)$ & & 48.7 & & \\
Vo & $(\mathrm{km} / \mathrm{s})$ & 0 & 50 & 0 & -50 \\
$\mathrm{~K}$ & $(\mathrm{~km} / \mathrm{s})$ & 68 & 72 & 107 & 78 \\
Eo & $(2,440,000+)$ & 5257.6 & 5258.0 & 5257.7 & 5258.6 \\
$\mathrm{f}(\mathrm{M})$ & $\left(\mathrm{M}_{\odot}\right)$ & 1.6 & 1.8 & 6.1 & 2.4 \\
\hline
\end{tabular}



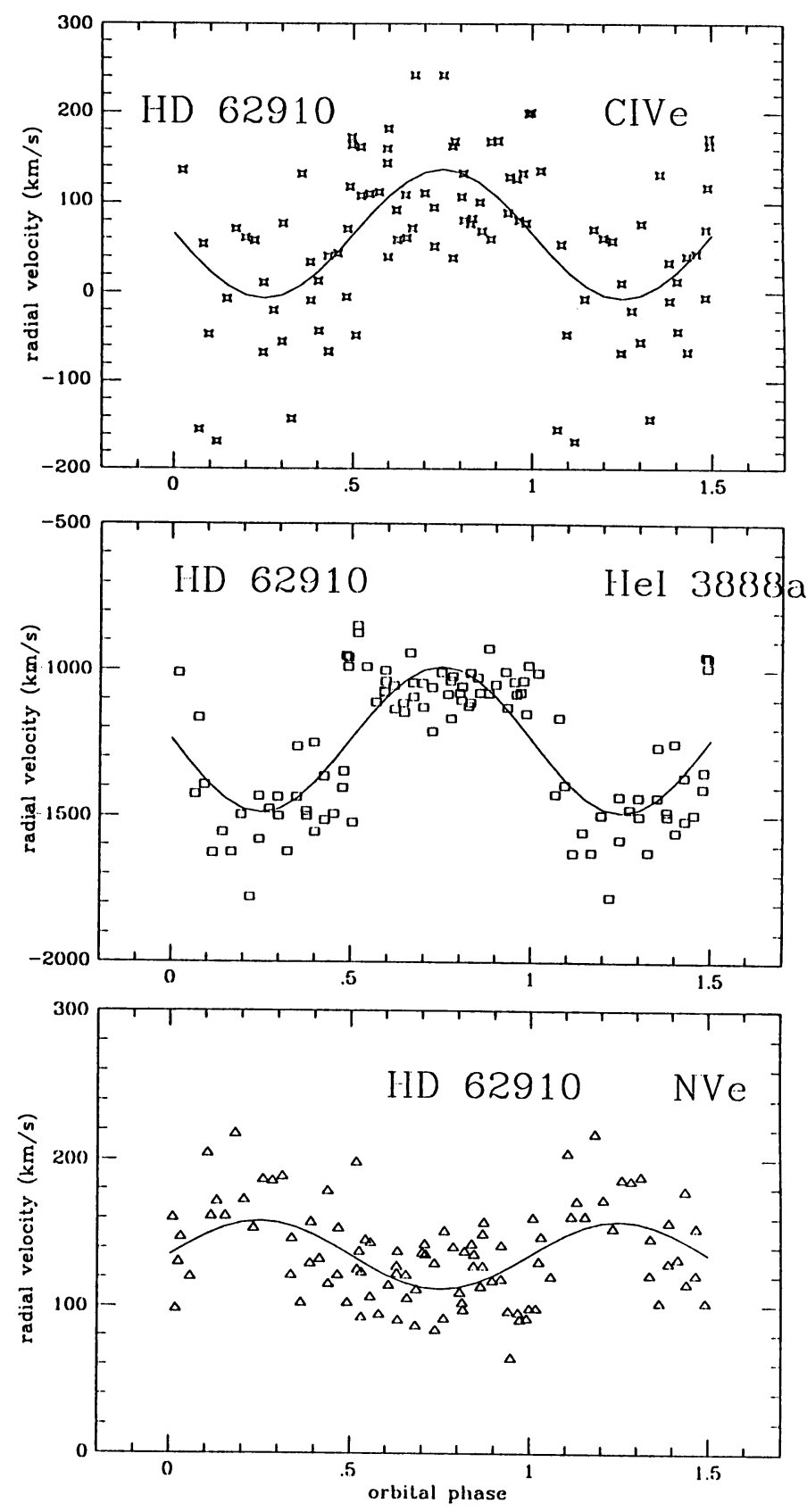

Figure 2. Observed radial velocities of HD 62910. The curves represent the radial velocity orbits from Table 2. 

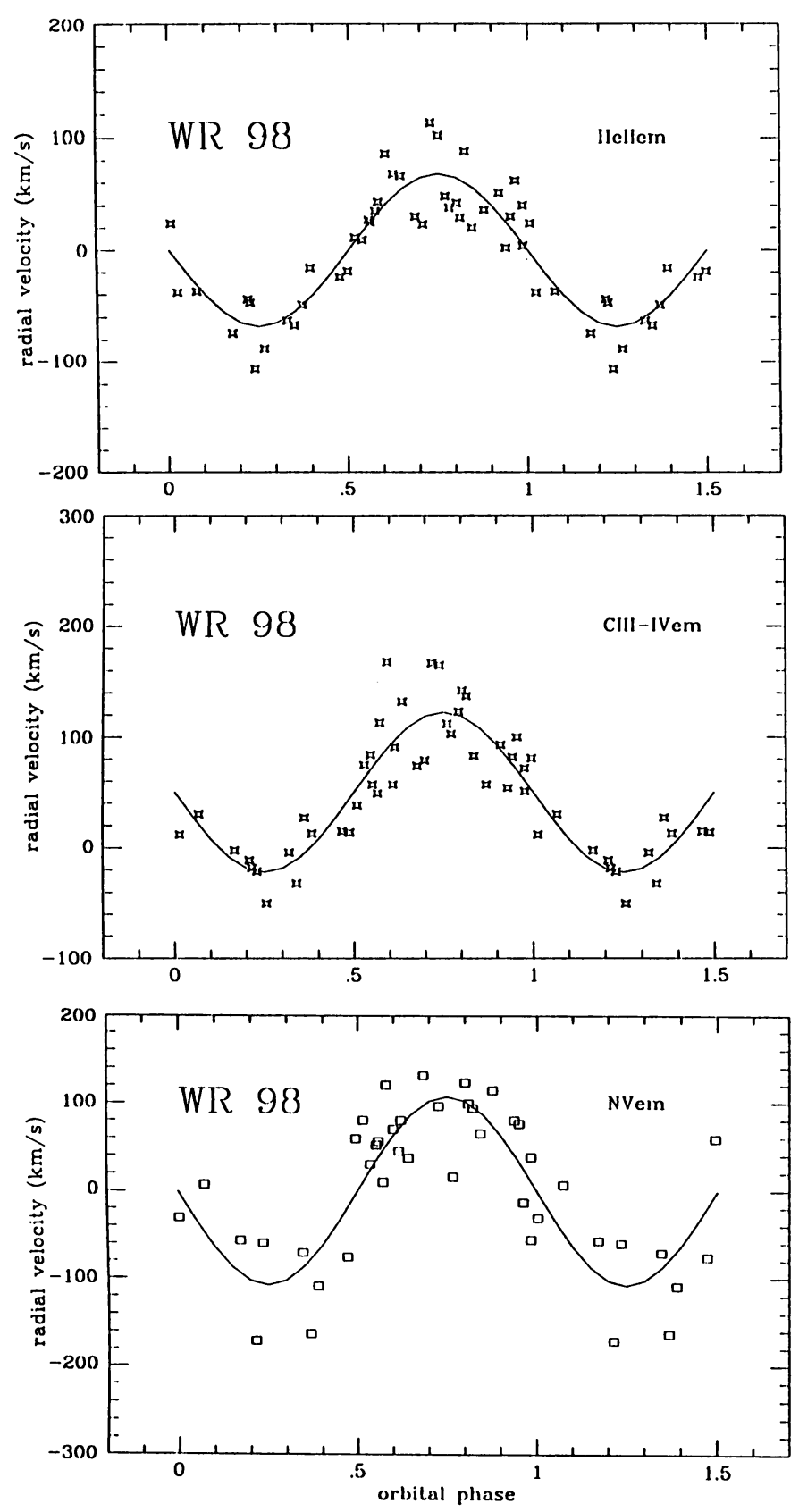

Figure 3. Observed radial velocities of WR 98. The curves represent the radial velocity orbits from Table 3 . 


\section{Conclusions}

Two new binary systems with WR components, namely Sk- $71^{\circ} 34$ and WR 98 have been discovered and another previously suspected binary, WR8, has been confirmed from extensive radial velocity studies. From the preliminary radial velocity orbit of $S k-71^{\circ} 34$, a WN3 + $\mathrm{O} 6$ binary in the Large Magellanic Cloud, rather large minimum masses are deduced for both components, about 18 and $28 \mathrm{M}_{\odot}$ for the WN3 and 06 , respectively.

The other two binaries, WR8 and WR98, are galactic stars both with WN+WC type spectra. WR98 is a single-lined binary, while WR8 appears to show $\mathrm{C}$ and $\mathrm{N}$ spectral features with antiphased motion. WN stars with strong carbon lines in their spectra are few in number. Only 8 are known among all the WR stars in our galaxy and the Magellanic Clouds (cf.Conti and Massey 1989). The binary nature of WR8 and WR98 brings to 5 the number of spectroscopic binaries among these WN/C stars, thus they may all be binaries.

Acknowledgements. This paper was prepared while the author was enjoying the hospitality of ESO at La Silla Observatory in the framework of their Senior Visitor programme. My atendance to the IAU Symposium No.143 was made possible thanks to generous grants from Fundacion Antorchas, Argentina; IAU, and the local organizing committee. I am much indebted to CTIO for the use of their facilities during many observing seasons.

\section{References}

Conti, P.S., and Garmany, C.D. 1983, Publ. Astron. Soc. Pacific, 95, 411.

Conti, P.S., and Massey, P. 1989, Astrophys. J., 337, 251.

van der Hucht, K., Conti,P.S., Lundstrom, I.,and Stenholm, B. 1981, Space Sci. Rev. 28, 227.

Niemela, V.S. 1982, in IAU Symp. No.99, C.de Loore and A.Willis, eds. p.299.

\section{DISCUSSION}

Koenigsberger: Do you see the $H e I 3888 \AA$ absorption actually moving or could the RV variations result from profile variations?

Niemela: I see it moving.

Conti: Concerning HD 69210, is the purported anti-phasing between the $C I V$ and $N V$ statistically significant?

Niemela: Yes.

Schmutz: It is difficult to understand that in a WN6+WC4 binary the P Cygni absorption of $\mathrm{HeI} \lambda 3888$ belongs to the WC star. "Normal" WC4 stars do not show observable $\mathrm{HeI}$ lines in the optical region.

Niemela: Many binaries do show the $\mathrm{HeI}$ absorption edges, even if the corresponding emission is not visible. On the other hand, HD 62910 is not a WC4 star.

Massey: For HD 62910, it would be useful to know if the correlation coefficient for $N V v s$. $C I V$ is statistically significant or not.

Niemela: It seems to be significant at the $90 \%$ level. 